\title{
EDITORIAL
}

\section{Implementation Science For Person-centered Dementia Care}

World-wide, there are over 47 million people living with dementia today (WHO, 2017). This number is expected to increase as the population ages because the neurodegenerative diseases which cause dementia, Alzheimer's Disease and vascular dementia, for example, are most common in those over the age of 65 .

People living with dementia have many challenges with respect to their health. Over the course of the disease trajectory most exhibit behavioral and psychological symptoms of dementia (BPSD) such as agitation, aggression and resistiveness to care. BPSD are burdensome to the person living with dementia as well as their family and formal caregivers. BPSD put people living with dementia at risk for inappropriate use of antipsychotic drugs that reduce their physical function, and increase risk for both social isolation and physical abuse (Kales et al., 2014). These challenges add to the high economic burden associated with neurodegenerative diseases (Hermann et al., 2006).

Behavioral approaches have repeatedly been endorsed by major geriatric organizations as the first line of treatment for BPSD (American Geriatrics Society..., 2003). Nurses are in a unique position to address BPSD because nonpharmacological approaches are the mainstay of the disciplines care. Nurse scientists have demonstrated that nonpharmacological approaches, such as leisure activities and assistance with activities of daily living, are effective for responding to BPSD (Kolanowski et al., 2011; Galik et al., 2014). Additionally, these approaches are most effective when delivered using person-centered principles of care. These principles include considering the persons preferences, interests and values in care planning and implementation.

Until recently, the major emphasis in nursing research has been on the development of new knowledge with limited focus on dissemination or implementation of effective approaches to BPSD in real world settings. The result is a gap between the percentage of individuals who could benefit from evidence-based non-pharmacological interventions and those who actually are exposed to these interventions.

Studies have shown that the effectiveness of evidence-based practices can be improved when delivered under conditions of an implementation framework. An international group of experts identified the testing of implementation strategies for addressing BPSD in nursing homes as a research priority (Morley et al., 2014). To move nursing science for BPSD forward, the next generation of nursing studies should address this pressing priority.

There are many implementation frameworks that can be used to guide the design of research studies. Nurse investigators who want to improve practice should become familiar with these frameworks and their use. An excellent overview is provided by Tabak and colleagues in a recent issue of the American Journal of Preventive Medicine (Tabak et al., 2012). At a very basic level, these frameworks direct the investigators' attention to complex multilevel contextual factors that affect the translation of effective and innovative approaches to practice: the inclusion of important stakeholders in the design and implementation of the research project; attention to the changing context in which care is provided; and the use of practical measures for assessing progress toward individually-established goals.

In the coming decades, every country will need to develop a plan for responding to the healthcare needs of its growing population of older adults who live with dementia. We know much about what constitutes quality dementia care, but less about how to implement it in practice settings. Implementation science holds promise for building the science of person-centered dementia care, and nurse scientists can provide leadership in using this science to improve practice.

Ann Kolanowski RN, PhD, FAAN

Professor of Nursing and Medicine

Colleges of Nursing and Medicine

Pennsylvania State University, USA 


\section{References}

American Geriatrics Society; American Association for Geriatric Psychiatry. The American Geriatrics Society and American Association for Geriatric Psychiatry recommendations for policies in support of quality mental health care in U.S. nursing homes. Journal of the American Geriatrics Society. 2003;51(9):1299-1304.

Galik E, Resnick B, Hammersla M, Brightwater J. Optimizing function and physical activity among nursing home residents with dementia: testing the impact of function-focused care. The Gerontologist. 2014;54(6):930-943.

Herrmann N, Lanctôt KL, Sambrook R, Lesnikova N, Hébert R, McCracken P, Robillard A, Nguyen E. The contribution of neuropsychiatric symptoms to the cost of dementia care. International Journal of Geriatric Psychiatry. 2006;21(10):972-976.

Kales HC, Gitlin LN, Lyketsos CG; Detroit Expert Panel on Assessment and Management of Neuropsychiatric Symptoms of Dementia. Management of neuropsychiatric symptoms of dementia in clinical settings: recommendations from a multidisciplinary expert panel. Journal of the American Geriatrics Society. 2014;62(4):762-769.

Kolanowski A, Litaker M, Buettner L, Moeller J, Costa PT jr. A randomized clinical trial of theory-based activities for the behavioral symptoms of dementia in nursing home residents. Journal of the American Geriatrics Society. 2011;59(6):1032-1041.

Morley J, Caplan G, Cesari M, Dong B, Flaherty JH, Grossberg GT, Holmerova I, Katz PR, Koopmans R, Little MO, Martin F, Orrell M, Ouslander J, Rantz M, Resnick B, Rolland Y, Tolson D, Woo J, Vellas B. International survey of nursing home research priorities. Journal of the American Medical Directors Association. 2014;15(5):309-312.

Tabak RG, Khoong EC, Chambers DA, Brownson RC. Bridging research and practice: models for dissemination and implementation research. American Journal of Preventive Medicine. 2012;43(3):337-350.

World Health Organization. Dementia. [cited 2017 Sep 19]. Available from: www.who.int/mediacentre/factsheets/fs 362/en/ 